Arterien. Wegen des hypertrophischen Zustandes der diese Gefäße auskleidenden Endothelien betrachtet Nauwerck die Sache als ein Angiom, welches aus hyperplastischen und hypertrophischen, größtenteils auch abnorm weiten KapillargefäBen zusammengesetzt ist.

Da aber über das Verhalten der Geschwulst zum Knochen, zu den Arterien des Oberschenkels etc. keine näheren Angaben vorliegen, der histologische Befund an der Geschwulst aber nach der gegebenen Schilderung und der Abbildung gewiß ein von unserer Beobachtung verschiedener war, so kann dieser Fall kaum mit Sicherheit als in die Gruppe der cirrsoiden Angiome gehöriger betrachtet werden.

Es bliebe demnach auch in obiger Hinsicht unser Casus ein bemerkenswertes Unikum.

Literatur.

v. Mikulicz-Kümmel, Handbuch der Krankheiten des Mundes, Jena 1898. Nauwerck, G., Dieses Archiv, Bd. 111.

Partsch, C., Erkrankungen der Mundhöhle, im Handbuch der praktischen Chirurgie.

\title{
XXII.
}

\section{Über einen Fall von Chorionepithelioma} der Harnblase.

(Aus dem Pathologisch-anatomischen Institut der Kaiserlichen Universität zu. Moskau.)

Von

Dr. W. St. Djewitzki.

Vor nicht allzu langer Zeit noch hatte es den Anschein, als ob die Frage über die Entstehung jener unter dem Namen "Chorionepithelioma" bekannten eigenartigen Geschwülste dank den Arbeiten unzähliger Autoren ziemlich genau erörtert sei; die Meinung, welche die Geschwülste. als einzig und allein den Frauen angehörig hinstellte, schien somit festen Fuß gefaßt zu haben. Nichtsdestoweniger wurden in den allerletzten Jahren 
neue Beobachtungen und Arbeiten veröffentlicht, welche die Tatsache feststellten, daß Geschwülste von identischem Bau anch dem Manne angehören können, bei demselben im Hoden vorkommen und einen gleichen bösartigen Verlauf zeigen. Im Hinblick darauf, daß solche Befunde den Gedanken an einen Zusammenhang solcher Tumoren mit einer Graviditas zerstörten, warf sich von selbst die Frage auf, ob solche Neubildungen überhaupt außerhalb einer Schwangerschaft bei weiblichen Individuen beobachtet wurden? Diese Frage muß ich in der Weise beantworten, daß eine ausführliche genaue Beschreibung solcher Fälle nicht existiert und daß folglich meine Beobachtung des vorliegenden Falles von Chorionepithelioma bei einer Virgo intacta als die erste und einzige dasteht. Ich fand zwar in der Literatur den Hinweis auf einen analogen Fall. Derselbe gehört Lubarsch an; doch letzterer erwähnt ihn nur flüchtig in einer seiner Arbeiten.

"Ich habe vor kurzem eine Geschwulst zur Untersuchung erhalten, die von dem Genitaltractus, wahrseheinlich dem Uterus eines 13 jährigen Mädchens stammte. Man hatte die Probelaparatomie gemacht; als sich aber ergab, daß die mächtigen Tumormassen, die vom Uterus auszugehen schienen, überall verwachsen waren, von der weiteren Operation Abstand genommen. Es wurde eine große Menge weicher blutreicher Tumormassen übersandt, die schon makroskopisch an die Chorionepitheliome erinnerten. Noch viel mehr tat das der mikroskopische Bau: es fanden sich große polygonale Zellen mit hellem Protoplasma und großen chromatinreichen Kernen, die sich zu Platten dicht aneinander legten, ferner große mit roten Blutkörperchen gefüllte Hohlräume, in die zottenartige, mit großkernigen und Riesenzellen ausgekleidete Gebilde hineinhingen usw. - Bevor ich wußte, daß der Tumor von einem 13jährigen Mädchen. stammte, hatte ich kaum einen Zweifel, daß es sich um ein Chorionepitheliom handeln würde. Da nun aber festgestellt wurde, daß der Tumor von einem Mädchen stammte, das virgo intakt war und überhaupt noch nicht menstruiert hatte, konnte daran nicht mehr gedacht werden. Da das Kind von den Angehörigen bald aus dem Krankenhaus genommen wurde und, nachdem es in seine Heimat (Russisch- 
Polen) zurücktransportiert war, jede Möglichkeit schwand, durch die Sektion genanere Aufklärung über den Ursprung des Tumors zu erhalten, ist der Fall nicht genügend geklärt. Jedenfalls beweist er aber auch so, daß das Bild des Chorionepithelioms auch ohne Bestehen einer Gravidität von Geweben des weiblichen Genitaltractus durch neoplastische Wucherung geliefert werden kann."

Indem ich mich mit dieser kurzen Bemerkung über diese rätselhafte und leider unaufgeklärte Beobachtung begnüge, wende ich mich zur Beschreibung meines eigenen Falles.

Am 1. Oktober des Jahres 1903 wurde die Patientin Nadjeschda M., ein Mädchen von 75 Jahren, mit Klagen über Blutungen, die zwei Jahre audauerten und sich besonders im Laufe der letzten drei Monate verstärkt hätten, auf die gynäkologische Klinik aufgenommen. Außerdem klagte Patientin über Dyspnoe, starken Husten mit Auswurf mit Blut und schmerzhaftes Urinieren.

Anamnesis. Die Menstrua stellten sich mit dem 18. Lebensjahre ein, erschienen alle drei Wochen mit einer Daner von 5-6 Tagen sehr profus. Sie sind seit 20 Jahren völlig erloschen. Eines Sistierens derselben kann sich Patientin nicht erinnern; sie ist Virgo. Vor zwei Jahren, nachdem schon längst die Climax begonnen hatte, stellten sich Blutungen ein. Seitdem wiederholten sich dieselben, doch nicht stark. Seit den großen Fasten des Jahres 1903 wurden sie häufiger und traten dabei so hochgradig auf, daß Patientin, schon äußerst geschwächt, sich einer Operation zu unterwerfen entschloB.

Status praesens. Patientin normal gebaut und gut genährt. Fettpolster gut ausgebildet. Bauch gewölbt; sein Umfang auf dem Nabelniveau $117 \mathrm{~cm}$. Bei Palpation desselben klagt Patientin über starke Schmerzen; eine Perkussion im. Bereiche des Colon descendens und über der Symphysis ergibt eine unbedeutende Dämpfung. Seitens des Verdauungstractus - schlechter Appetit, beständige Verstopfung. Hämorrhoiden - abs.

In Leber und Milz nichts Abnormes.

Atmungsorgane: Starke Dyspnoe sowohl bei Bewegung als auch im Ruhezustand; Husten mit reichlichgem Auswurf und Blut. Atmung unregelmäßig oberflächlich, 42 pro Minute im Ruhezustand. Lungengrenze hinten gesunken auf eine Rippenbreite. Lungenschall überall sonor. Auscultation: Sowohl vorne wie hinten viele Rasselgeräusche, besonders hinten unten - oedematös.

Herz: Grenzen normal. Auscultation: Geränsch mit dem zweiten Ton. Puls regelmäßig, 92 pro Minute. Zeitweise Herzklopfen. Gefäße derb.

Harnblase schmerzhaft beim Druck. Urinieren schmerzhaft; Drang gesteigert. Urin von rötlicher Färbung, trübe, mit reichlichem Nieder- 
schlag. Spezifisches Gewicht 1,021. Eiweiß 5 pro mille. Reaktion neutral. Tagesquantum $600 \mathrm{ccm}$. Die mikroskopische Untersuchung konnte kein Nierenepithel entdecken. In der Klinik wurde Schneiden beim Urinieren konstatiert. Urinmenge sank bis $300,200,100 \mathrm{kcm}$.

Genitalien: Brüste gut entwickelt, etwas hart, schmerzhaft. Brustwarzen deutlich. Warzenhof nicht pigmentiert. Becken normal. Inguinaldrüsen nicht palpierbar. Große Labien oedematös; kleine und Clitoris gerötet. Offnung der Urethra geschwollen. Damm normal. Hymen unversehrt. Vaginalwände glatt, von elastischer Konsistenz; Empfindlichkeit nicht gesteigert. Scheidengewölbe ausgeglichen. Sekretion unbedeutend.

Von seiten des Zentralnervensystems -- guter Schlaf. Überall Hyperästhesie der Haut.

Auf Grund der klinischen Diagnose Fibromyoma uteri interstitiale und im Hinblick auf die Schwäche und das hohe Alter der Patientin wurde beschlossen, von einer radikalen Operation abzustehen und sich zur Erzielung einer Blutstillung mit einer Abrasio des Cavum uteri zu begnügen. Am 8. Oktober wurde unter leichter Chloroformnarkose mit einer vorhergegangenen Morphiuminjektion die Operation (Hymenschnitt und Abrasio) vollzogen. Beim Sondieren betrug die Länge der Uterushöhle $15 \mathrm{~cm}$.

Im weiteren Verlaufe des Krankheit stellten sich Sypmtome geschwächter Herzaktion ein, welche ihren Ausdruck in der Verminderung der Urinmenge, Oedem des ganzen Körpers und Gesichts, starker Đyspnoe mit Husten und Blutauswurf fanden. Die Temperatur war die ganze Zeit hindurch ungefähr normal, nicht höher als 37,50. Puls 80-160 pro Minute.

Am 19. Oktober erfolgte unter progressiver Herzschwäche und Schlafsucht der Exitus letalis.

Bevor ich jedoch zum Sektionsprotokolle übergehe, berichte ich hier über die Befunde, welche Privatdozent H. Greife bei der mikroskopischen Untersuchung der Abrasionsstïckchen erhielt, und die er mir freundlichst überlassen hat. Dem geehrten Herm Kollegen statte ich zugleich meinen aufrichtigsten Dank ab. Die Ergebnisse der Untersuchung waren - folgende: Die Drüsenzahl ist vergrößert; die Drüsen selbst sind verlängert und erscheinen in Art von verzweigten und gewundenen Schläzchen; ihr Lumen ist stellenweise erweitert. Von seiten des interstitieellen Gewebes lassen sich keine erwähnungswerten Veränderungen hervorheben. Im allgemeinen das Bild einer Endometritis glandularis hyperplastica et hypertrophica.

Die Autopsie führte ich im Pathologisch-anatomischen Institute am 20. Oktober 1903 aus.

Sektionsprotokoll. Die Leiche des alten Mädchens ist gut gebaut und genährt. Hautfarbe bleich. Haut des Bauches gespannt, glatt; keine Spuren einer stattgehabten Graviditas in Art von Striae gravidarum. Fettpolster stark ausgebildet. Das Zwerchfell steht rechts auf der Höhe der 4., links der 5. Rippe. Lungen sehr fest mit den Brustkastenwänden 
verwachsen. Ihre Pleura bedeutend verdickt, von weißlicher Farbe, erscheint in Art einer die ganze Lunge umgebenden und beim Herausnehmen dieser letzteren zerrissenen Membran. Die Langen sehr massiv, ihre Oberfläche uneben, höckerig; bei der Palpation erweist sich das Lungenparenchym durchsetzt von Knoten der Gröbe einer Walnuß entsprechend und kleiner von ziemlich fester Konsistenz. Auf der durch die ganze Lunge führenden Schnittfläche treten diese Knoten bedeutend über der Oberfläche des Lungengewebes hervor, zeigen eine dunkelrote Färbung und erscheinen gleichsam spongiös. Im Zentrum einiger größerer Knoten läßt sich Gewebszerfall nachweisen. Das fast bis auf ein Minimum reduzierte Langenparenchym erscheint etwas ödematös und hyperämisch. Die Schleimhaut der Bronchien, mit dunkelbraunem Sekret bedeckt, ist unbedeutend geschwollen, von dunkelroter Farbe. Bronchialdrüsen vergrößert, derb, auf der Schnittfläche anthrakotisch und von derselben dunkelroten Masse durchsetzt, wie die Lungenknoten.

Im Herzbeutel etwa ein halbes Glas klarer gelblicher Flüssigkeit. Herz reichlich mit Fett bedeckt. Herzmuskel schlaff; die Ventrikel nicht kontrahjert. Unter dem Endokard haben die Muskeln das Aussehen eines Tigerfells; auf der Schnittfläche von gelblicher Lehmfarbe. Endokard und Klappen unverändert. Koronararterien starr, zeigen ein deutliches Lumen. Intima der Aorta mit zerstreuten sklerotischen Platten bedeckt.

Milz nicht vergröBert. Kapsel glatt; am hinteren Milzrande entdeckt man ein etwas hervortretendes Knötchen von dunkelroter Farbe, $1 \mathrm{~cm}$ im Durchmesser. Was das Milzgewebe betrifft, so bietet es keine Veränderungen.

Leber nicht vergrößert. Die obere Leberoberfläche glatt. Acinöse Zeichnung undeutlich; Neubildungsknoten nicht vorhanden.

Gallenblas e von vier groBen Steinen ausgefïllt; dieselben sind von bräunlicher Farbe, viereckig geformt und facettiert.

Nieren: Mäßige Verdichtung des Parenchyms, bedeutende Sklerose und Starrheit der Gefäße; Verdünnung der Kortikal- und Medullarsubstanz und reichliche Fettablagerung im Nierenbecken.

Die Ureteren unverändert.

Die Harnblase ist an der Symphyse gelegen und ans der Höhlung des kleinen Beckens durch den vergrößerten fibromyomatösen Uterus verdrängt. Sie erscheint in kontrahiertem Zustande und ist mit reichlichem Fettgewebe bedeckt. Als Inhalt eine geringe Menge trüben Urins. Auf der hinteren Mucosafläche entdeckt man eine Neubildung, regelmäßigrundlich geformt, $5 \mathrm{~cm}$ im Durchmesser, etwas über der Oberfläche der übrigen Schleimhaut hervortretend, von dunkelroter Färbung mit einer unebenen, ulcerösen, von Salzen inkrustierten Oberfläche. Die unmittelbar daran grenzende Schleimhautpartie zeigt im Vergleich zur übrigen grauweißen unveränderten Mukosa der Harnblase eine rote Färbung.

Uterus bedeutend vergrößert, füllt fast das ganze kleine Becken aus. Seine Vergrößerung verdankt er hauptsächlich den zahlreichen, ziem- 
lich umfangreichen, bis hühnereigroßen, derben, weißlichen, faserigen, mit einer gleichmäßigen glatten Oberfläche versehenen Knoten. Dieselben sind subserös, intermuskulär und submukös gelegen. Uterushöhle ist verlüngert und erweitert. Der Kanal verläuft nicht in gerader Richtung infolge der hervortretenden oben beschriebenen Knoten. Die Innenfläche des Cavum uteri ist mit einem bräunlichen zähen Sekret bedeckt; nach Entfernung desselben erscheint sie glatt, rosa gefärbt.

Die Eierstöcke sind klein und bieten Erscheinungen einer senilen Atrophie.

Tuben unverändert.

Vagina und äußere Genitalien zeigen entsprechend den klinischen Erscheinungen keine Veränderungen.

An der Magenschleimhaut lassen sich einige kleine, rundlich geformte, hämorrhagische Erosionen mit schwarzem Grund konstatieren.

Von seiten der Darmschleimhaut ist nichts Bedeutsames zu erwähnen außer einem kleinen, rötlichen Knötchen von der Größe einer kleinen Erbse in der Flexura sigmoides.

Omentum und überhaupt das subseröse Gewebe enthalten bedeutende Fettlager. Mesenterialdrüsen sind unverändert.

Diagnosis anatomica: Neoplasma vesicae urinariae. Cystitis. Neoplasma secundarium pulmonum, lienis et intestini. Pleuritis chronica adhaesiva bilateralis. Degeneratio adiposa. myocardii. Atrophia levis renum arteriosclerotica. Degeneratio parenchymatosa hepatis. Cholelithiasis. Myofibromata uteri multiplices. Erosiones ventriculi haemorrhagicae. Adipositas universalis.

Einer mikroskopischen Untersuchung unterlagen Stückchen aus verschiedenen Stellen der Harnblase, der Uterusknoten und der metastatischen Knoten der Lunge, der Bronchialdrüsen, der Milz und des Darms. Fixiert wurden dieselben in vierprozentiger Formalinlösung. Die mit Haematein-Eosin gefärbten -Celloidinpräparate zeigten die weitêr unten beschriebenen Bilder einer mehr oder weniger gleichmäßig gebauten Neubildung.

Harnblase. Das Gewebe der Neubildung bestand durchweg aus zelligen Elementen zweier Typen ohne jegliche Bindegewebsgrundlage, doch untermischt mit einer bedeutenden Menge von Blutelementen und Fibrinzügen. Es prävalierten Zellen von rundlicher polygonaler Form mit einem großen bläschenartigen Kern. Die Kerne waren gleichfalls rund oder oval, mit einem spärlichen Chromatinnetz versehen und enthielten meist einen Nucleolus; vom umgebenden Protoplasma waren sie durch eine deutlich ausgedrïckte Kernmembran getrennt. Das Protoplasma war gleichsam durchsichtig und nahm von Eosin eine nur blasse Färbung 
an. Diese Zellen lagen gewöhnlich dicht aneinandergedrängt und erzeugten das Aussehen von Epithelschichten. Die Grenzen zwischen solchen Zellen traten deutlich hervor. Der andere Zelltypus zeichnete sich durch den Reichtum seines Protoplasma und die Menge der Kerne in demselben aus; dabei waren ihnen äuberst wunderliche Formen und eine oft merkwürdige Verlagerung der Bestandteile eigen. Die Kerne solcher Zellen waren von gedehnter, länglicher Form, chromatinreich und nahmen eine intensive Hamateinfärbung an. Das Protoplasma schien homogen und färbte sich gat mit Eosin; an vielen Zellen sah man in demselben kleinere und größere Vakuolen, welche meist leer waren, manchmal jedoch grat erhaltene rote Blutkörperchen aufwiesen. Hinsichtlich des Verhaltens dieser Zellen weiter za den oben beschriebenen (Zellen des. I. Typas) komte man folgendes beobachten: bald sah man sie ohne jegliche Ordnung inmitten jener Zellen verlagert; bald schien es, als ob sie dieselben umaingelten, indem ihr Leib gedehnt und geschlängelt sich den Formen jener Epithelschichten anpaBte; endich trafen sich auch solehe von unregelmäßig randlicher Form; das Protoplasma derselben zeigte fast gar keine Ausläufer, und diese Zellen lagen in Art von Klümpchen nnter den übrigen. Entsprechend dem beschriebenen Zustande des Protoplasma lagen auch die Kerne solcher Zellen entweder nebeneinander, einer dem anderen folgend, oder sie waren aufeinandergeworfen und erschienen dann in Art von Chromatinhaufen. Von grobem Interesse sind das Verhalten und, wie es scheint, eine gewisse tendenziöse Neigung dieser Zellen zi den Blathöhlen und Extravasaten. An den einen Stellen umgaben sie von allen Seiten eine Bluthöhle, sich in schmale zirkuläre Protoplasmastreifen mit langgezogenen Kernen ausdehnend; an anderen grenzten sie nur von einer Seite an die Blutansammlung, halbmondförmig, mit dünnen protoplasmatischen Auslaufern versehen und mit einigen Kernen im zentralen Teile; weiterhin lagen diese Riesenzellen stellenweise frei in groben Blathöhlen, wo sie wunderliche Formen annahmen und Ausläufer besaßen, durch welche sie miteinander verbunden erschienen; hie und da sah man zwischen solchen Zellen auch Blut und eine kömige rosabläuliche Masse. Kurz, es entstanden äuBerst hübsche, verschiedenartige Bilder, die zu beschreiben ein Ding der Unmöglichkeit ist.

Um das Strukturbild dieser Neubildung zu vollenden, mub ich noch eine Art von Zellen erwähnen, die ich, wenn auch nicht äberall, doch an vielen Stellen entdecken konnte; nämlich solche, die meiner Ansicht nach den Ubergang zwischen jenen beiden Zelltypen bildeten. Von den Zellen des I. Typus anterschieden sie sich durch eine intensivere Färbung des Kernchromatins; ein Netz konnte man in dem Kern schon nicht mehr konstatieren. Die Form der Kerne entsprach entweder vollständig jenen, oder sie waren mehr den Riesenzellenkernen ähnlich, indem sie gedehnt, ungleichmäbig gebogen und in vielen Zellen zu mehreren aufraten. Was das Protoplasma betrifft, so war es nicht so durehsichtig, wie bei jenen ersten Zellen, auch nicht so homogen und valkolisiert, 'wie bei den 
Zellen des zweiten Typus. Stellenweise lagen solche Zellen so dicht, daß sich eine Grenze zwischen ihnen nicht erkennen ließ, and dam trugen sie das Aussehen von Riesenzellen, indem sie sich von den wirklichen, ich möchte sagen, nur durch eine geringere Protoplasmamenge unterschieden. Wie ich schon bemerkte, befanden sich inmitten solcher typisehen zelligen Elemente rote und weiße Blutkörperchen, Fibrin und mehr der Peripherie zu eine Menge Chromatinkörnchen, ein koaguliertes Gewebe mit kaum bemerkbaren Spruren seiner Struktur, endlich Kalkteilchen.

An der inneren Grenze stieb man auf ein progressives Durchwachsen durch das Muskelgewebe der Harnblase. Hier befanden sich hauptsächlich Zellen des I. Typus, welche die Muskelfasern von allen Seiten umwuchsen und in die Muskelsubstanz hineindrangen. Es verbreiteten sich diese Zellen anch in dem intermuskulären Bindegewebe, wobei ihre Kerne nicht selten Kerrteilungsfiguren zeigten. Sich den Gefäßen nähernd, umwuchsen diese Zellen die Wandungen derselben; in der Umgebung waren daselbst oft Blutextravasate zn konstatieren, welche, wie es schien, auf eine Zerstörung der Gefäßwandungen hinwiesen. In vielen Gefäßlumina lagen, sie bald vollständig bald nur zum Teil ausfüllend, Zellen beider Typen, teils vermischt mit noch erhaltenen Blutelementen, teils inmitten von eigenartigen homogenen thrombotischen Massen.

An der Hamblasenschleimhaut konnte eine fast totale Abwesenheit des Epithels und eine starke Blutüberfüllung der kleinen GefäBe konstatiert werden. An manchen Stellen ließ sich in Blutgefäßen die beginnende Entwicklung von metastatischen Knoten nachweisen; in der Nitte oder zur Seite fand sich ein GefäB, ringsumher im Crewebe eine hämorrhagische Infiltration und inmitten der letzteren einige einkernige Zellen; in umfangreicheren Knoten traten dann auch Riesenzellen auf.

Die mikroskopische Untersuchung der im Uterus gefundenen Knoten an Stückchen, welche der Innenfiäche des Cavum nteri entnommen waren, ergab ein äußerst einfaches, der makroskopischen Diagnose entsprechendes Bild: die Schleimhant fehlte; die äußerste Gewebsschicht bestand aus oedematösem, teilweise nekrotischem, von roten Blatkörperchen durchtränktem. Bindegewebe; die daselbst befindichen GefäBe waren hochgradig mit Blut gefiullt, teils thrombiert. Das Knotengewebe bildeten Stränge aus glatten Muskelfasern, die nach verschiedenen Richtungen hin verliefen und durch dünne Bindegewebsschichten voneinander getrennt waren; stellenweise trafen sich nekrotische Partien fibromyomatösen Gewebes. Eine genaue Untersuchung komte keine Spuren der in der Harublase gefundenen Zellen entdecken.

Die Lnngenknoten waren von demselben Ban wie das Neubildungsgewebe der Harnblase. Das nmgebende Lnngengewebe erschien komprimiert, hyperämisch und oedematös; in den Gefäßen befanden sich stellenweise dieselben Neubilangszellen.

Die Bronchialdrüsen waren fast durchweg von demselben Nenbildungsgewebe ersetzt oder bestanden ans umfangreichen Blutansamm- 
Jungen mit Fibrinzügen und hier and da verlagerten wunderlichen Riesenzellen. An der Peripherie ließen sich stellenweis Reste des lymphoiden Gewebes nachweisen, und in den zentralen Partien zeugten die noch inmitten der Neubildungszellen erhaltenen Kohleteilchen von der einst vorhandenen Drüse.

Das Gewebe der metastatischen Knoten in der Milz und dem Dickdarm zeigte die oben beschriebenen Bestandteile and wies in seinem Bau keine neuen Befunde auf, die einer besonderen Beschreibung bedürtten.

Die bei der mikroskopischen Untersuchung gewonnenen Befunde ergaben dermaßen das typische Bild einer Neubildung sui generis, wie man es am wenigsten in dem gegebenen Falle erwarten konnte. Die beiden bescbriebenen Zelltypen hinterließen keinen Zweifel darüber, daß die einen Zellen den sogenannten Langhansschen Zellen, die anderen dem Syncytium entsprachen, und daß hier im Hinblick auf ihren Zusammenhang, ihre vasodestruierende Tätigkeit, ihre Bösartigkeit, für welche die zahlreichen Metastasen in der Blutbahn deutlich sprachen, die Rede nur von dem sogenannten Chorionepithelioma sein kann. Die bei der Sektion wegen des charakteristischen, ich möchte sagen spezifischen, makroskopischen Aussehens und besonders wegen der Knoten im Lungengewebe ausgesprochene Voraussetzung eines solchen Baues dieser Neubildung fand zur großen Verwunderung in dem mikroskopischen Bilde ihre Bestätigung. Doeh erschien in gegebenem Falle die Voraussetzung eines solchen Tumors am Sektionstische wenig zulässig und widersprach der gegenwärtigen Anschauung über die Entstehung des Chorionepithelioma.

Es ist schon seit lange festgestellt, daß das Chorionepithelioma bei Frauen unbedingt im Zusammenhang mit einer Graviditas steht and sich im Uterus und den Adnexen nach Geburten, Aborten und Blasenmolen entwickelt, wobei sich ein enger genetischer Zusammenhang mit diesen pathologischen Zuständen konstatieren läßt, da die Neubildung von den embryonalen Zottenepithelien ausgeht. Spätere Untersuchungen bestätigten diese Befunde und gingen noch weiter. Es sind Fälle beschrieben worden, die eine gewisse Analogie mit dem meinigen zulassen, and wo im Uterus und in den Adnexen bei der Sektion und mit Hilfe des Mikroskops keine Spur von 
einem Chorionepithelioma entdeckt wurde, während nicht selten die äußeren Genitalien und andere Organe eine große Anzahl der beschriebenen Knoten aufwiesen, welche letztere bei der mikroskopischen Untersuchung das allbekannte Bild dieses Tumors zeigten. Ich weise hiermit anf die letzten, teils ähnlichen Arbeiten hin: Zagoranski-Kissel - 16 Fälle, Hugo Hübel - 8 Fälle, Busse - 2 Fälle. Obwohl diese interessanten Fälle eine ganze Literatur geschaffen haben, so können sie doch keine Verwunderung noch Zweifel weder von klinischem noch pathologiseh-anatomischem Standpunkte aus hervorrufen. Vom ersteren wiederum deswegen, weil sie bei schwanger gewesenen Franen beobachtet wurden, wobei die Graviditas gewöhnlich abnorm verlanfen war; vom zweiten Standpunkte aus erklären sie sich durch einen Eintritt, eine retrograde Embolie der losgelösten Teilchen des Zottenepithels, der Zotten selbst oder eines gewöhnlichen Embolus in die BlutgefäBe der Mutter, - - mit anderen Worten, wir beobachten hier die Erscheinungen jenes Prozesses, welcher unter dem Namen „Parenchymzellenembolie" bekannt ist und auch bei einer normalen Graviditas stattfinden kann. Diese Fälle sind natürlich von wichtiger klinischer Bedeutung und veranlassen die Gynäkologen ( $\mathrm{Hübl}$ ), die Chorionepitheliome in zwei Gruppen zu trennen: 1. Chorionepithelioma uteri et adnexorum and 2. Chorionepithelioma raginae et vulvae bei vollständig gesunden Genitalien.

Bei einer Zusammenstellung dieser Angaben über das Chorionepithelioma mit den Befunden meines Falles ergibt sich eine Identität nur in der Struktur und höchstens noch darin, dab auch in meinem Falle alle Genitalien frei von einem ähnlichen Tumor waren. Was jedoch die Entstehung des beschriebenen Tumors, d. $h$. seine Abhängigkeit von der Eizelle nud das entsprechende klinische Bild betrifft, so steht unser Fall in völligem Widerspruch zu der oben besprochenen Anschanung über das Chorionepithelioma. Dieser Widerspruch zeigt sich in der unwiderruflichen Tatsache, daß die Patientin eine Virgo intacta war, also niemals schwanger gewesen ist, folglich also auch in keinerlei Beziehung za den pathologischen Prozessen einer Graviditas stehen konnte. Somit ist eine Mög- 
lichkeit, die Entstehung dieser Geschwulst vom Standpunkte jener bestimmten, schon lange festgestellten Anschauung zu erklären, vollständig ausgeschlossen.

Wenn wir alles dies in Betracht nehmen, so wird es klar, daß der beschriebene Fall ganz einzig dasteht und daß seine Entstehung wahrscheinlich auf anderen Faktoren beruht. Der Fall bleibt doch ungeachtet einer sorgfältigen und genauen. Untersuchung rätselhaft. Die Sache ist die, daß er als einziger in der Literatur, zugleich dermaßen weit in seiner Entwicklung vorgeschritten war, daß es äußerst schwer, ja selbst unmöglich ist, sich hinsichtlich seiner Entstehung auszusprechen.

Dennoch will ich mich bemühen, an der Hand der Literaturangaben einer Lösung dieser schwierigen Frage näher zu treten. Unsere Geschwulst könnte man vor allen Dingen den sogenannten "Sarcomes angioplastiques". gleichstellen, welche im Jahre 1878 von Malassez und Monod beschrieben wurden, da sie ein denselben identisches Strukturbild zeigt und gleichzeitig nicht mit einer Graviditas in Zusammenhang steht. Irgendwelche histogenetische Befunde, auf Grund welcher man auf diese Weise die Geschwulst ansprechen könnte, ergab die Untersuchung nicht, dazu steht die Frage über die Geschwulst "Sarcome angioplastique" auf einem Standpunkte, nach welchem die neueren Autoren eine Existenz derselben sehr bezweifeln. Die Fälle, in welchen ein dem Chorionepithelioma ähnliches Gewebe beobachtet wurde, werden von den neueren Autoren als Teratome traktiert, dabei wird von allen angenommen, daß in embryoiden Geschwülsten sich ein dem Chorionepithelioma, natürlich nur histogenetisch identisches Gewebe entwickeln kann.

Der oben beschriebene Bau unserer Geschwulst liefert keine Befunde für eine Lösung der Frage in diesem Sinne. Folglich ist eine Möglichkeit, dieselbe für ein Teratom zu erklären und auf die eine oder andere Weise ihrer Entstehung näher zu treten, ausgeschlossen, wenn man sich natürlich nicht der etwas eigenartigen, doch wenig begründeten Meinung anschließen will, die von Pick ausgesprochen wurde. Dieser Autor drückt sich in folgender Weise aus: "Das Chorionepithelioma oder die traubenmolenartigen Produkte können als Bestandteile der Teratome, sei es allein, oder in Begleitung von anderen Ge- 
websarten, vorkommen außer jeglichem Zusammenhang mit einer Graviditas und zwar in allen Teilen des Organismus sowohl des Mannes, als auch der Frau." Seine Ansicht, daß in den Teratomen sich auch nur ein bestimmtes Gewebe entwickeln kann, bestärkt er mit dem Falle von Saxer, der einen isolierten Zahn im Eierstockgewebe beschrieben hat; doch Pick läßt dabei folgenden Umstand außer acht, nämlich daß die Wandung der Zahnhülle aus einer Bindegewebsschicht mit zahlreichen Lymphspalten bestand und daß die Innenfläche derselben mit einem dünnen vielreihigen Epithel aus flachen Zellen ausgekleidet war, $\mathrm{d}$. $\mathrm{h}$. es war ein gewöhnliches Teratom mit den Abkömmlingen verschiedener Keimblätter.

Mit dieser Meinung Picks in Einverständnis zu kommen, oder ihr irgendwelche Bedeutung zu schenken, scheint mir nicht wohl möglich. Wenn es sich wirklich so verhielte, so könnte man auch ein Lipom oder Sarkom in gewissen Fällen nach Belieben für die Bestandteile eines Teratoms ansehen, welches in einem nur mehr entwickelten Gewebe seinen Ausdruck fand. Dann würde auch die Geschwulstlehre bedeutend vereinfacht werden und zu einem so merkwürdigen Satze zusammenfallen, wie: alle Geschwülste stellen entweder völlig entwickelte Teratome oder nur Teile derselben vor, je nach der Zahl der sie zusammensetzenden Gewebe.

Indem ich also keine bestimmten Befunde weder selbst besitze, noch in der Literatur vorfinde, die eine Entstehung dieser Geschwulst erklären könnten, möchte ich doch zwei mögliche Voraussetzungen nicht außer acht lassen.

Erstens, wenn wir die im Uterus gefundenen fibromatösen Knoten in Betracht nehmen, so können wir folgender Erwägung Raum geben: Wir wissen, daß die gegenwärtige Ansicht hinsichtlich einer kongenitalen Entstehung der Fibromyome des Uterus als höchst wahrscheinlich erscheint; diese Tatsache, welche anf eine abnorme Entwicklung der Genitalien im embryonalen Zustande hinweist, muß die mögliche Voraussetzung einer gewissen Abnormität in der Entwicklung der benachbarten Organe, also auch der Harnblase, bestärken. Wenn wir weiter in Betracht nehmen, daß unsere Geschwulst auf der hinteren Wand der Harnblase saß, und uns der Entwicklung dieses Organs 
erinnern, so stellt es sich heraus, daß gerade hier an der hinteren Wand im zweiten Monat des embryonalen Lebens die Mündungen der Wolfschen Gänge (Disse) zu liegen kommen. Von diesem Standpunkte ausgehend und die Möglichkeit zulassend, daß in der Wand der entwickelten Harnblase möglicherweise die Elemente des Wolfschen Ganges geblieben waren, mit welchen auch die ektodermalen Zellen der Cloake mitgeschleppt waren, da der W.-Gang (nach Kollmann) in gewisser Entwicklungsperiode einen Kontakt mit dem Ektoderm, vielleicht auch eine Verschmelzung vollzieht, kann man, wie es mir scheint, in den letzteren, mitgeschleppten Zellen den Beginn dieser Geschwulst sehen, welche, wie bekanntlich, auch als Produkt des undifferenzierten ektodermalen Chorionepithels hingestellt wird.

Andererseits kann man die Möglichkeit einer Entstehung dieser Geschwulst aus dem Epithel der Harnblase nicht völlig von der Hand weisen. Weder für noch wider läßt sich etwas sagen, wiederum weil die Geschwulst an der Stelle ihres primitiven Herdes schon zu weit in ihrer Entwicklung vorgerückt ist. Die Frage, ob eine solche „Rückbildung" des Harnblasenepithels in embryonales Epithel (nach Ribbert) oder „Entdifferenzierung" Lubarschs möglich ist, harrt somit noch ihrer Lösung.

Zum Schluß ist es mir eine angenehme Pflicht, meinem hochverehrten Liehrer, dem Herrn Professor M. Nikifor off, für die freundlichen Anweisungen meinen aufrichtigsten Dank auszusprechen.

\section{Literatur.}

1. Münzer: Chorionepithelioma malignum. Centralblatt für Allgem. Patholog. u. pathol. Anat. Bd. XIII, Nr. 6-7.

2. Nikifor off: Uber die sogenannten bösartigen Deciduome. Podwyssotzkis Archiv Bd. I, 1896.

3. Wlassow: Zur Lehre von den embryoiden Geschwälsten. Dissert. Moskau 1903.

4. Steinert: Uber die embryoid. Geschwülste der Keimdrüsen. Dieses Arch. Bd. 174, Heft 2, 1903.

5. Lubarsch: Die Metaplasiefrage. Arbeiten aus der path-anat. Abteilung des Hygien. Inst. zu Posen. 1901. 
6. Zagoranski-Kissel: Ober das primäre Chorionepithelioma. Arch. für Gynäk. Bd. 67 (zitiert nach Busse).

7. Busse: Uber Chorionepithelioma. Dieses Arch. Bd. 174, Heft 2, 1903.

8. Hübl: Uber das Chorionepithelioma in der Vagina. Wien 1903.

9. Pick: Zur Kenntnis der Teratome. Berlin, Kl. Wochenschrift Nr. 51, 1902.

10. Snjegirjoff: Uterusblutungen.

11. Saxer: Beiträge zur Pathol. Anatomie. Ziegler, Bd. 31, 1902.

12. Disse: Handbuch der Anatomie des Menschen. Bd. VII, Teil I, 1902. Herausg. v. Bardeleben.

13. Kollmann: Lehrbuch der Entwicklungsgeschichte des Menschen. Jena 1898, S. 402.

\section{XXIII. \\ Über das Schicksal der in die Blutbahn gebrachten Purinkörper.}

Von

W. Ebstein und E. Bendix.

(Hierzu Tafel XV.)

Die vorliegende Arbeit bezweckt das Schicksal einer Reihe von in die Blutbahn gebrachten Substanzen bezüglich ihrer Ausscheidung zu verfolgen. In erster Reihe interessierten uns die im tierischen Organismus vorkommenden Purinkörper.

I. Harnsäureversuche.

In einer experimentellen Arbeit hat der eine von uns (Ebstein) in Gemeinschaft mit A. Nicolaier ${ }^{1}$ die Ausscheidung der Harnsäure durch die Nieren genauer studiert. .In dieser Arbeit kam es vornehmlich darauf an, zu ermitteln, wie sich bei dem Tierversuche die Verhältnisse gestalten, wenn in die Blutbahn eines Tieres, welches unter nơrmalen Verhältnissen nur Spuren von Harnsäure bildet, verhältnismäßig g r o B e Mengen dieser Substanz gebracht werden. Die Verfasser hofften dabei eine Steinbildung erzielen zu können; doch erwies sich diese Hoffnung als trügerisch. Aber es wurde in diesen Versuchen gezeigt, in welcher Weise die Versuchstiere (Kaninchen) bestrebt sind, diese ihrem Organismus fremde Substanz so schnell wie möglich und so viel wir es damals übersahen, wenigstens zu 